\title{
Numerical solution of the discrete-time, convergent, non-negative definite Lyapunov equation
}

\author{
Sven Hammarling \\ Numerical Algorithms Group Ltd, Wilkinson House, Jordan Hill \\ Road, Oxford OX2 8DR, UK
}

Received 8 March 1991

Abstract: An algorithm for the numerical solution of the real discrete-time non-negative definite Lyapunov equation is discussed. An improved rank 2 updating formula is proposed for the Cholesky factor that arises during the numerical solution.

Keywords: Discrete Lyapunov equation; Cholesky factors.

\section{Introduction}

The real, discrete-time, convergent, non-negative definite Lyapunov equation is of the form

$A^{\mathrm{T}} X A-X=-B^{\mathrm{T}} B$

where $A$ is an $n$ by $n$ convergent matrix, so that the eigenvalues satisfy $\left|\lambda_{i}\right|<1$, and $B$ is an $m$ by $n$ matrix. In this case $X$ is non-negative definite and hence possesses a Cholesky factorization

$X=V^{\mathrm{T}} V$,

where $V$ is upper triangular. The aim of the method to be discussed here is to determine the Cholesky factor $V$, without forming $B^{\mathrm{T}} B$ and without first solving for $X$, this aim being motivated by considerations of numerical stability. The method is an extension of the Bartels-Stewart method [1], first described by Hammarling [4] for both the continuous and discrete-time cases. An improved algorithm is given here for the real discrete-time case, that also corrects one of the formulae in the original algorithm ${ }^{l}$. This version, together with the algorithm for the continuous case, has been

${ }^{1}$ Equation (10.22) in [4]. successfully used in the SLICE Library [8] since 1986 and subsequently in the SLICOT Library [6], which has superseded SLICE. A correction for the formula has also been noted more recently by Varga [7], but we believe that the improvement given here is preferable.

In the next section we recap on the method for solving the Lyapunov equation. Here we only give a brief summary of the method, further details can be found in [4] and background information in Golub and Van Loan [3]. In Section 3 we look at the details of the new rank 2 update.

\section{Solution of the Lyapunov equation}

Let the Schur factorization of $A$ be

$A=Q S Q^{\mathrm{T}}$,

where $Q$ is orthogonal and $S$ is block upper triangular, with one by one and two by two diagonal blocks, each two by two block corresponding to a complex conjugate pair of eigenvalues of $A$. Then equation (1) can be transformed to the form

$S^{\mathrm{T}}\left(U^{\mathrm{T}} U\right) S-U^{\mathrm{T}} U=-R^{\mathrm{T}} R$,

where $U$ is the Cholesky factor of $\tilde{X}=Q^{\mathrm{T}} X Q$ and $R$ is the Cholesky factor of $Q^{\mathrm{T}}\left(B^{\mathrm{T}} B\right) Q$. Note that we can obtain $R$ from a $Q R$ factorization of $B Q$ and similarly, once $U$ has been found, $V$ can be obtained from a $Q R$ factorization of $U Q^{\mathrm{T}}$.

If we partition $S, U$ and $R$ conformally as

$$
\begin{aligned}
& S=\left(\begin{array}{cc}
s_{11} & s^{\mathrm{T}} \\
0 & S_{1}
\end{array}\right), \quad U=\left(\begin{array}{cc}
u_{11} & u^{\mathrm{T}} \\
0 & U_{1}
\end{array}\right), \\
& R=\left(\begin{array}{cc}
r_{11} & r^{\mathrm{T}} \\
0 & R_{1}
\end{array}\right),
\end{aligned}
$$


where $s_{11}$ is either a scalar, or a two by two matrix, then equation (4) leads to the equations

$s_{11}^{\mathrm{T}}\left(u_{11}^{\mathrm{\top}} u_{11}\right) s_{11}-u_{11}^{\mathrm{\top}} u_{11}=-r_{11}^{\mathrm{\top}} r_{11}$,

$S_{1}^{\mathrm{T}} u \beta-u=-r \alpha-s u_{11}^{\mathrm{T}} \beta$,

$\alpha=r_{11} u_{11}^{-1}, \quad \beta=u_{11} s_{11} u_{11}^{-1}$.

$S_{1}^{\mathrm{T}}\left(U_{1}^{\mathrm{T}} U_{1}\right) S_{1}-U_{1}^{\mathrm{T}} U_{1}=-\hat{R}^{\mathrm{T}} \hat{R}$,

where

$\hat{R}^{\mathrm{T}} \hat{R}=R_{1}^{\mathrm{T}} R_{1}+y y^{\mathrm{T}}$,

$y y^{\mathrm{T}}=r r^{\mathrm{T}}-u u^{\mathrm{T}}+v v^{\mathrm{T}}$,

$v=S_{1}^{\mathrm{T}} u+s u_{11}^{\mathrm{T}}$.

By considering the Cholesky factorization of $R_{1}^{\mathrm{T}} R_{1}$ $+y y^{\mathrm{T}}$ we see that we can take $\hat{R}$ to be upper triangular. When $s_{11}$ is a one by one block so that $s_{11}=\lambda_{1}$ then, with some algebraic manipulation, the equations simplify to

$\left(\lambda_{1}^{2}-1\right) u_{11}^{2}=r_{11}^{2}$,

$\left(\lambda_{1} S_{1}^{\mathrm{T}}-I\right) u=\alpha r-\lambda_{1} u_{11} s$,

$S_{1}^{\mathrm{T}}\left(U_{1}^{\mathrm{T}} U_{1}\right) S_{1}-U_{1}^{\mathrm{T}} U_{1}=-\hat{R}^{\mathrm{T}} \hat{R}$,

where

$$
\begin{aligned}
& \hat{R}^{\mathrm{T}} \hat{R}=R_{1}^{\mathrm{T}} R_{1}+y y^{\mathrm{T}}, \\
& y=\alpha v-\lambda_{1} r, \quad v=S_{1}^{\mathrm{T}} u+u_{11} s .
\end{aligned}
$$

We can readily see that equation (8) can be solved for $u_{11}$, equation (9) can then be solved for the vector $u$ and equation (10) is a reduced order Lyapunov equation. The Cholesky factor $\hat{R}$ can be found by performing the $Q R$ factorization

$$
\left(\begin{array}{l}
R_{1} \\
y^{\mathbf{T}}
\end{array}\right)=\hat{Q}\left(\begin{array}{l}
\hat{R} \\
0
\end{array}\right)
$$

a straightforward rank 1 update of the Cholesky factor $R_{1}$.

When $s_{11}$ is a two by two matrix then, in principle, we have the same situation for equations (5) and (6), but we have to take care of some tricky numerical details, to ensure numerical stability, in solving for the two by two matrix $u_{11}$ and the two column matrix $u$, including the case where $u_{11}$ is singular, and these details are discussed in Hammarling [4]. In the next section we show how to obtain $\hat{R}$ as a rank 2 update to $R_{1}$, in an analogous manner to the above rank 1 case.

\section{The rank 2 update}

Firstly we note that equation (4) can be written as

$U^{\mathrm{T}} U=\left(\begin{array}{c}R \\ U S\end{array}\right)^{\mathrm{T}}\left(\begin{array}{c}R \\ U S\end{array}\right)$

and that

$\left(\begin{array}{c}R \\ U S\end{array}\right)=\left(\begin{array}{cc}r_{11} & r^{\mathrm{T}} \\ 0 & R_{1} \\ u_{11} s_{11} & v^{\mathrm{T}} \\ 0 & U_{1} S_{1}\end{array}\right)$

Now perform the $Q R$ factorization

$\left(\begin{array}{cc}r_{11} & r^{\mathrm{T}} \\ u_{11} s_{11} & v^{T}\end{array}\right)=\tilde{Q}\left(\begin{array}{cc}t_{11} & p^{\mathrm{T}} \\ 0 & z^{\mathrm{T}}\end{array}\right)$.

Equation (12) then becomes

$\left(\begin{array}{cc}u_{11}^{\mathrm{T}} u_{11} & u_{11}^{\mathrm{T}} u^{\mathrm{T}} \\ u u_{11} & u u^{\mathrm{T}}+U_{1}^{\mathrm{T}} U_{1}\end{array}\right)$
$=\left(\begin{array}{cc}t_{11}^{\mathrm{T}} t_{11} & t_{11}^{\mathrm{T}} p^{\mathrm{T}} \\ p t_{11} & p p^{\mathrm{T}}+R_{1}^{\mathrm{T}} R_{1}+z z^{\mathrm{T}}+S_{1}^{\mathrm{T}} U_{1}^{\mathrm{T}} U_{1} S_{1}\end{array}\right)$

which gives the three equations

$u_{11}^{\mathrm{T}} u_{11}=t_{11}^{\mathrm{T}} t_{11}$,

$u u_{11}=p t_{11}$,

$u u^{\mathrm{T}}+U_{1}^{\mathrm{T}} U_{1}=p p^{\mathrm{T}}+R_{1}^{\mathrm{T}} R_{1}+z z^{\mathrm{T}}+S_{1}^{\mathrm{T}} U_{1}^{\mathrm{T}} U_{1} S_{1}$.

The second of these equations implies that $u=$ $p t_{11} u_{11}^{-1}$ and hence, using the first, we get

$u u^{\mathrm{T}}=p t_{11} u_{11}^{-1} u_{11}^{-\mathrm{T}} t_{11}^{\mathrm{T}} p^{\mathrm{T}}=p p^{\mathrm{T}}$

and thus the third equation gives

$S_{1}^{\mathrm{T}}\left(U_{1}^{\mathrm{T}} U_{1}\right) S_{1}-U_{1}^{\mathrm{T}} U_{1}=-\left(R_{1}^{\mathrm{T}} R_{1}+z z^{\mathrm{T}}\right)$,

so that $z \equiv y$ is the required rank 2 update for $R_{1}$. In practice, in place of equation (13), we perform the equivalent $Q R$ factorization

$\left(\begin{array}{cc}\alpha & r^{\mathrm{T}} \\ \beta & v^{\mathrm{T}}\end{array}\right)=\tilde{Q}\left(\begin{array}{ll}\tau & p^{\mathrm{T}} \\ 0 & y^{\mathrm{T}}\end{array}\right), \quad \tau=t_{11} u_{11}^{-1}$, 
and from equation (5) we can see that $\alpha^{\mathrm{T}} \alpha+\beta^{\mathrm{T}} \beta$ $=I$, so that

$\tau^{\mathrm{T}} \tau=I$.

We use this form of the $Q R$ factorization because, when $u_{11}$ is singular, we can still define $\alpha$ and $\beta$ (see Hammarling [4], sections 6 and 10) and, for these, equation (3) and the important identity $u u^{\mathrm{T}}=p p^{\mathrm{T}}$ are still true. Having found $y$ we then, of course, obtain the Cholesky factor $\hat{R}$ from the $Q R$ factorization of equation (11), where now $y^{T}$ contains two rows.

\section{Conclusion}

We have looked at the numerical solution of the real, discrete-time, convergent, non-negative definite Lyapunov equation, concentrating particularly on the case where the Schur factor of $A$ has a two by two block, corresponding to a complex conjugate pair of eigenvalues of $A$. The algorithm discussed here, together with the continuous analogue, has been successfully implemented in the SLICOT control engineering library [6], and used in applications such as balanced state-space realization $[5,2]$.

\section{References}

[1] R.H. Bartels and G.W. Stewart, Solution of the matrix equation $A X+X B=C$, Comm. Assoc. Comput. Mach. 15 (1972) 820-826.

[2] K.V. Fernando and S. Hammarling, A product induced singular value decomposition (IISVD) for two matrices and balanced realization, in: B.N. Datta, C.R. Johnson, M.A. Kaashoek, R.J. Plemmons, and E.D. Sontag, Eds., Linear Algebra in Signals, Systems, and Control (SIAM, PA, Philadelphia, 1988) 128-140.

[3] G.H. Golub and C.F. Van Loan, Matrix Computations (Johns Hopkins University Press, Baltimore, MD, 2nd edition, 1989).

[4] S. Hammarling, Numerical solution of the stable, nonnegative definite Lyapunov equation, IMA J. Numer. Anal. 2 (1982) 303-323.

[5] A.J. Laub, M.T. Heath, C.C. Paige and R.C. Ward, Computation of system balancing transformations and other applications of simultaneous diagonalization algorithms, IEEE Trans. Automat. Control 32 (1987) 115-122.

[6] NAG and WGS, SLICOT Library, Release 1. Technical Report TR4/90, Numerical Algorithms Group, Wilkinson House, Jordan Hill Road, Oxford OX2 8DR, 1990. Also published as WGS-Report 90-1.

[7] A. Varga, A note on Hammarling's algorithm for the discrete Lyapunov equation, Systems Control Lett. 15 (1990) 273-275.

[8] T.W.C. Williams, Numerically reliable software for control: The SLICE Library, IEE Proc. Part D 133 (1986) 73-82. 